\title{
Case Report: Diagnosis of Dual-Biopsy Negative Severe Cardiac Amyloidosis
}

\author{
Kristin Lohr, MD \\ Thomas Jefferson University, kristin.lohr@jefferson.edu \\ Eric Kasprowicz, MD \\ Thomas Jefferson University, eric.kasprowicz@jefferson.edu
}

Follow this and additional works at: https://jdc.jefferson.edu/tmf

Part of the Cardiology Commons, and the Internal Medicine Commons

Let us know how access to this document benefits you

\section{Recommended Citation}

Lohr, MD, Kristin and Kasprowicz, MD, Eric (2016) "Case Report: Diagnosis of Dual-Biopsy Negative Severe Cardiac Amyloidosis," The Medicine Forum: Vol. 17 , Article 6.

DOI: https://doi.org/10.29046/TMF.017.1.007

Available at: https://jdc.jefferson.edu/tmf/vol17/iss1/6

This Article is brought to you for free and open access by the Jefferson Digital Commons. The Jefferson Digital Commons is a service of Thomas Jefferson University's Center for Teaching and Learning (CTL). The Commons is a showcase for Jefferson books and journals, peer-reviewed scholarly publications, unique historical collections from the University archives, and teaching tools. The Jefferson Digital Commons allows researchers and interested readers anywhere in the world to learn about and keep up to date with Jefferson scholarship. This article has been accepted for inclusion in The Medicine Forum by an authorized administrator of the Jefferson Digital Commons. For more information, please contact: JeffersonDigitalCommons@jefferson.edu. 


\section{Case Report: Diagnosis of Dual-Biopsy Negative Severe Cardiac Amyloidosis}

Kristin Lohr, MD and Eric Kasprowicz, MD

\section{INTRODUCTION}

Amyloidosis is a rare disorder that involves the deposition of misfolded protein in extracellular tissue. Disease manifestations vary depending on the affected organs. Areas most often involved include the kidneys, gastrointestinal tract, heart, nervous system, and musculoskeletal system. The most common type is AL amyloidosis, in which plasma cells produce an abundance of a monoclonal proteins that affect numerous organ systems. A second variety, AA amyloidosis, is associated with inflammatory diseases such as rheumatoid arthritis and inflammatory bowel disease. Finally, a heritable version of amyloidosis mediated by mutations in the transthyretin protein (TTR) has a predilection for deposition in peripheral nerves and cardiac tissue

\section{CASE PRESENTATION}

A 72 year-old female with a history of non-ischemic cardiomyopathy (NICM) with an ejection fraction (EF) of 30-35\%, monoclonal gammopathy of undetermined significance (MGUS), and chronic kidney disease (CKD) Stage 4 presented to the ED with several weeks of intermittent dizziness, nausea with dry heaving, intermittent shortness of breath, and pleuritic chest pain. At the time of admission she denied exertional chest pain, lower extremity swelling, or recent weight gain. She endorsed stable four-pillow orthopnea, good urine output in response to her home dose of bumetanide, and adherence to all her medications as well as to a low salt diet.

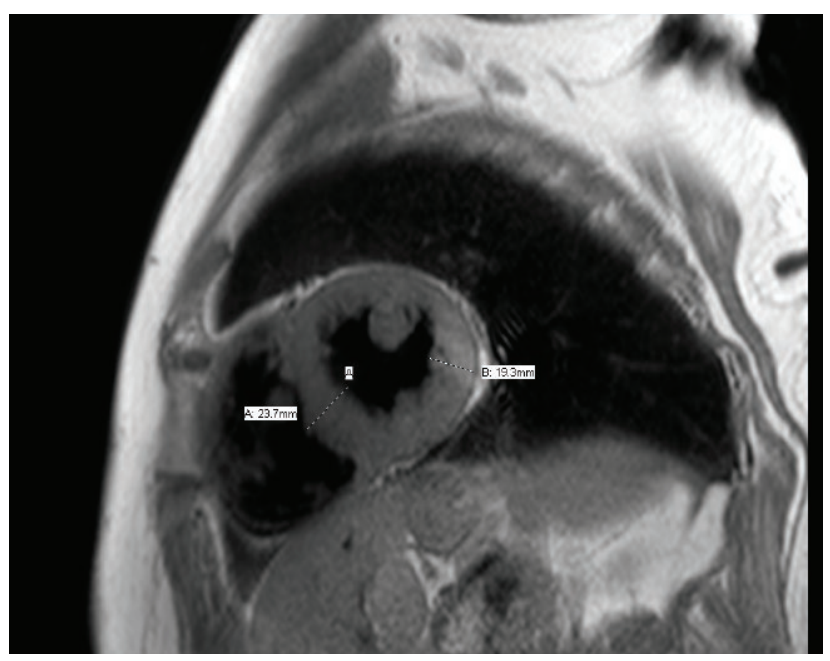

Figure 1. Cardiac MRI
The patient was diagnosed with NICM in 2013 after presenting with rapidly progressive dyspnea on exertion, new lower extremity swelling, and five-pillow orthopnea. A CT of her thorax at that time showed hilar lymphadenopathy, and transthoracic echocardiogram demonstrated an EF of $30 \%$, decreased from $45 \%$ in 2012. A cardiac catheterization in 2012 revealed no coronary artery disease, and, given her new hilar lymphadenopathy on imaging, an infiltrative process such as sarcoidosis or amyloidosis was suspected as a possible cause for her worsening heart failure. She underwent a fat pad biopsy at the time which was negative for amyloidosis. To follow, a cardiac MRI revealed no definitive evidence of sarcoidosis or amyloidosis. Furthermore, the patchy, delayed mid-myocardial enhancement in the left ventricle was attributed to viral myocarditis and thus the likely cause for her new onset heart failure (Figure 1). After diagnosis, the patient's EF recovered to $65 \%$ with aggressive management, but declined again to 30-35\% despite optimal therapy. Transthoracic echocardiogram from this admission demonstrated an ejection fraction of $35 \%$, biventricular hypertrophy with a restrictive pattern, and biatrial enlargement (Figure 2).

Other relevant medical history includes an ablation for atrial tachycardia six months prior to this admission. She also had a history of Protein S Deficiency complicated by pulmonary embolism requiring anticoagulation on warfarin.

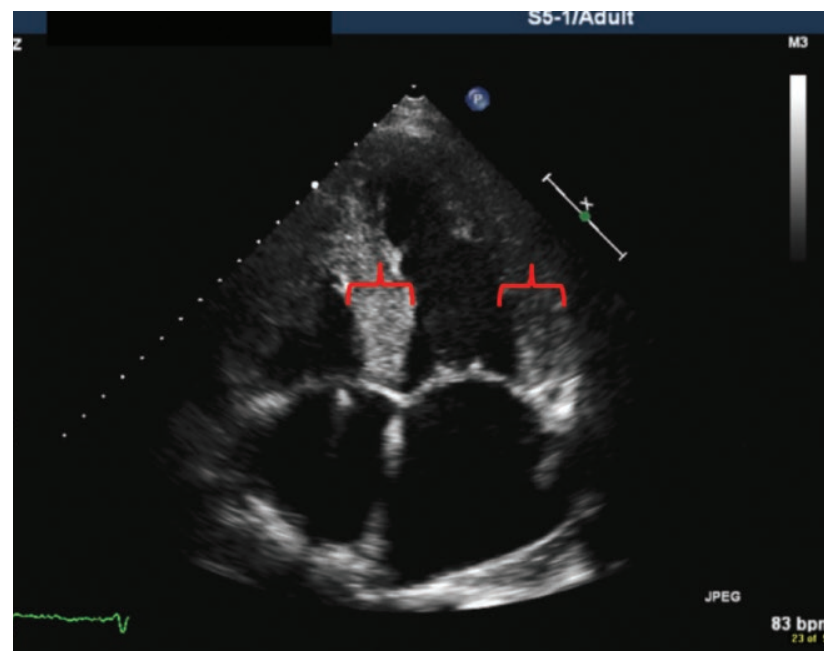

Figure 2. Four-chamber view of the patient's echocardiogram, displaying ventricular wall thickening and granular appearing myocardium. 


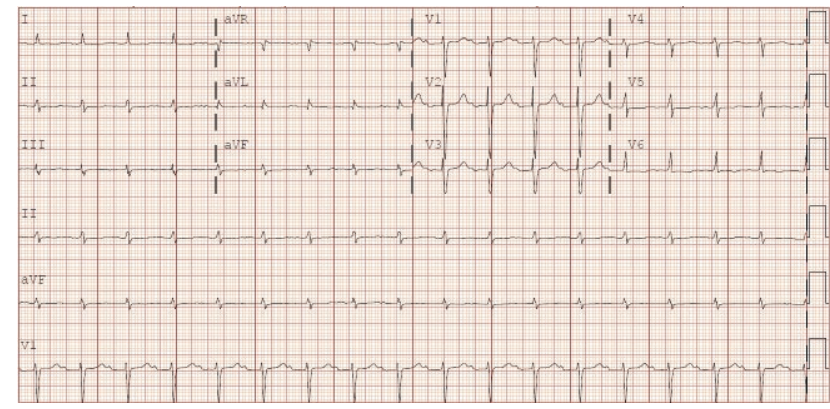

Figure 3. Electrocardiogram findings from our patient demonstrating low voltage in the limb leads and 1st degree AV block

Physical examination on admission revealed normal vital signs, lungs clear to auscultation with no crackles, cardiac exam with normal S1/S2 sounds, a tachycardic rate with regular rhythm and no murmurs, rubs, or gallops. Pulses were normal and JVD was not present. Extremities were warm, well perfused and without any peripheral edema. Labs were significant for a troponin elevation to $0.21 \mathrm{ng} / \mathrm{mL}$ (baseline $0.15 \mathrm{ng} / \mathrm{mL}$ ) and pro-brain natriuretic peptide (pro-BNP) elevated to $10,358 \mathrm{pg} / \mathrm{mL}$ compared to $4,700 \mathrm{pg} / \mathrm{mL}$ at her last admission 6 months earlier. EKG showed normal sinus rhythm with first-degree AV block and low voltage (Figure 3 ), and chest $x$-ray showed no consolidations or pulmonary edema.

\section{DIFFERENTIAL DIAGNOSIS}

The patient's presenting symptoms were attributed to an exacerbation of her chronic systolic heart failure, and she was admitted for diuresis and further management. Despite this initial assessment and her elevated pro-BNP, she appeared euvolemic on subsequent examination with no elevation of jugular venous pressure, no peripheral edema, and clear lungs.

With this ambiguous clinical picture, a right heart catheterization was performed to assess her hemodynamics. The catheterization revealed a pulmonary capillary wedge pressure of $15 \mathrm{~mm} \mathrm{Hg}$, right atrial pressure of $18 \mathrm{~mm} \mathrm{Hg}$, and cardiac index of $1.6 \mathrm{~L} /$ $\mathrm{min} / \mathrm{m}^{2}$ consistent with low output heart failure.

Despite her negative fat pad biopsy in the past, other clinical data (echocardiogram, EKG, past medical history, and physical examination) supported a diagnosis of amyloidosis. Given the continued suspicion, an endomyocardial biopsy was performed at the time of her right heart catheterization and stained positive for Congo Red, consistent with a diagnosis of amyloidosis (Figure 4). Further typing by mass spectrometry was performed at the Mayo Clinic Laboratory which showed hereditary ATTR amyloidosis with a Val122lle mutation.
A)

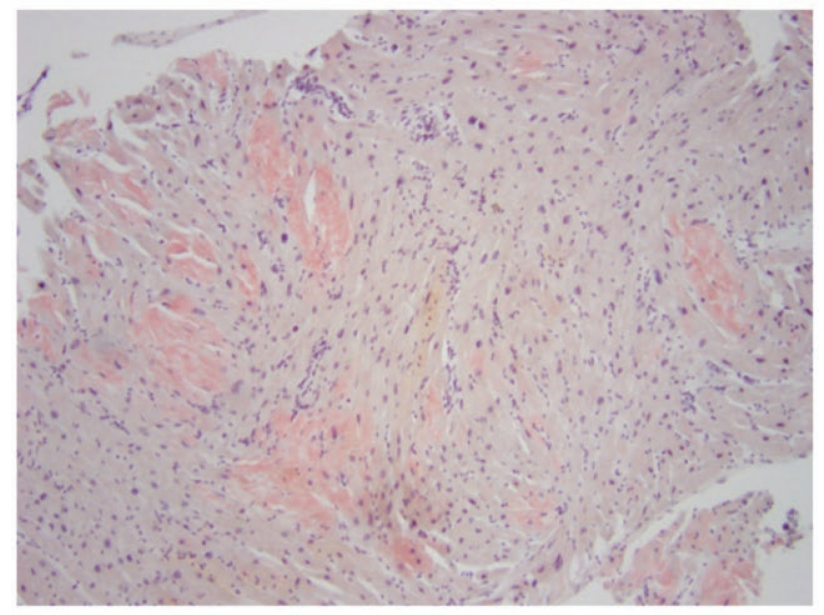

B)

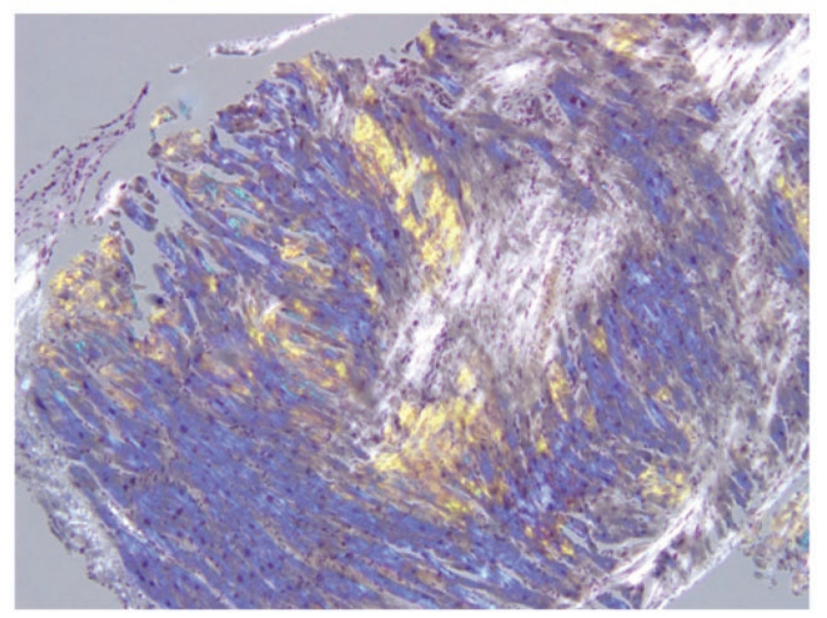

Figure 4. Endomyocardial biopsy slides from this case stained with Congo Red. (A) In this figure the normal myocardium is off-white, and the areas of amyloid deposition are salmon-colored (Congo red stain; Bright field microscopy, 10X magnification). (B) Demonstrates Congo-red staining under a polarized filter. In this figure, normal myocardium appears blue, collagen (fibrosis) is white, and amyloid deposits are yellow-green (Congo red stain; Polarized light microscopy, 10X magnification).

\section{OUTCOME AND FOLLOW-UP}

The patient continues to follow up with Cardiology, Nephrology and Hematology/Oncology for management of her cardiac amyloidosis. She has remained stable on her medication regimen since discharge. Further management decisions regarding her amyloidosis were deferred at time of discharge to await the results of mass spectrometry. Unfortunately, ATTR amyloidosis does not respond to chemotherapy as AL amyloidosis may, and supportive care is the only management in cases ineligible for transplant². Typically, liver transplantation is used to remove the culprit organ for abnormal TTR synthesis, and heart transplant may be used in management of end stage heart failure secondary to 
cardiac amyloid 2,3 . Given her advanced age and need for combined liver-heart transplantation, she is not a candidate for these traditional therapies but may be eligible for clinical trials of small molecular inhibitors for ATTR amyloid ${ }^{4}$

\section{DISCUSSION}

The diagnosis of cardiac amyloid is suggested by increased ventricular wall thickness and granular appearance of myocardium on echocardiogram (Figure 2) as well as low voltage on EKG (Figure 3). Tachyarrhythmias and prolonged PR and QT intervals are also often associated with cardiac amyloidosis, both of which were present in this patient. As mentioned above, the patient had first-degree A/V block and a prolonged QT interval on admission (Figure 3), in addition to recent ablation for atrial tachycardia.

Cardiac MRI is helpful in identifying cardiac amyloidosis but is not very sensitive in the early stages of the disease? This was likely the case in our patient, as the MRI was performed around the time of the patient's initial presentation of heart failure. The gold standard for diagnosis is endomyocardial biopsy (Figure 3), but this is not typically a first line diagnostic tool given the ease of obtaining biopsy samples from other sites, such as the abdominal fat pad or bone marrow. Interestingly, abdominal fat pad and bone marrow biopsies will only verify the diagnosis of amyloidosis in $40-80 \%$ of cases $^{5}$. This is particularly true in the Val122lle mutation seen in our patient, where amyloid deposition is primarily confined to the heart?

Cardiac amyloidosis is among the most serious manifestations of amyloidosis and is associated with poor prognosis and high mortality. Median survival from diagnosis of this variant of ATTR amyloidosis is approximately 2-3 years ${ }^{1}$. Once the diagnosis is made, management centers around supportive care and medical management of heart failure symptoms. Additionally, the underlying protein misfolding disorder should be treated, if possible, with chemotherapy in the case of AL amyloid and with liver transplant in the case of ATTR amyloid ${ }^{1}$. As mentioned above, these patients are sometimes evaluated for dual liver-heart transplantation depending on the severity of their disease ${ }^{3}$.

\section{KEY POINTS}

This case illustrates a presentation of cardiac amyloid with many classic diagnostic findings. These include: EKG findings of first degree A/V block and low-voltage complexes, atrial tachyarrhythmia, and elevated pro-BNP without clear signs of overt volume overload. Unfortunately for this patient, her prognosis remains poor, but the case illustrates an important point - in cases of progressive non-ischemic heart failure with an unclear etiology, it is important to keep a broad differential and consider cardiac amyloid if clinical signs point toward the diagnosis. Biopsies from non-cardiac tissue can provide important diagnostic information, possibly confirming the disease, but lack the sensitivity needed to completely rule out the disorder. Endomyocardial biopsy remains the gold standard for diagnosis of cardiac amyloid and should be pursued if clinical suspicion for the disease remains high.

\section{REFERENCES}

1. Patel KS, Hawkins PN. Cardiac Amyloidosis: Where are we today? Journal of Internal Medicine. 2015; 278: 126-44

2. Kapoor P, Thenappan T, Singh E, Kumar S, Greipp P. Cardiac Amyloidosis: A Practical Approach to Diagnosis and Management. The American Journal of Medicine. 2011; 124: 1006-1015

3. Pilato E, Dell'Amore A, Botta L, Arpesella G. Combined Heart and Liver Transplantation for Familial Amyloidotic Neuropathy. European Journal of Cardiothoracic Surgery. 2007; 32(1): 180-182

4. Sekijima, Y. Transthyretin (ATTR) Amyloidosis: Clinical Spectrum, Molecular Pathogenesis and Disease-Modifying Treatments. Journal of Neurology, Neurosurgery and Psychiatry. 2015; 86: 1036-1043

5. Gertz MA, Dispenzieri A, Sher T. Pathophysiology and Treatment of Cardiac Amyloidosis. Nature Reviews Cardiology. 2015; 12(2): 91-102 